\title{
Increased corpus callosum volume in children with chromosome 22q11.2 deletion syndrome is associated with neurocognitive deficits and genetic polymorphisms
}

\author{
Vandana Shashi ${ }^{\star, 1,2}$, Alan Francis ${ }^{3}$, Stephen R Hooper ${ }^{4}$, Peter G Kranz ${ }^{5}$, Michael Zapadka ${ }^{6}$, Kelly Schoch ${ }^{1}$, \\ Edward $\mathrm{Ip}^{7}$, Neeraj Tandon ${ }^{3}$, Timothy D Howard ${ }^{2}$ and Matcheri S Keshavan ${ }^{3}$
}

Chromosome 22q11.2 deletion syndrome (22q11DS) is associated with neurocognitive impairments. The neural substrates of cognitive impairments in 22q11DS remain poorly understood. Because the corpus callosum (CC) is found to be abnormal in a variety of neurodevelopmental disorders, we obtained volumetric measurements of the $\mathrm{CC}$ and its subregions, examined the relationship between these regions and neurocognition and selected genotypes within candidate genes in the $22 \mathrm{q} 11.2$ interval in 59 children with 22q11DS and 53 control subjects. The total CC, splenium and genu were significantly larger in children with 22q11DS and the enlargement was associated with better neurocognitive functioning in the 22q11DS group, suggestive of a compensatory increase in the CC volumes. The expected age-related increase in the volume of the CC was not seen in children with 22q11DS, indicative of dysmaturation of the $C C$ in these children. The increased volumes in the genu, splenium and total CC in the 22q11DS group were associated with polymorphisms within the candidate genes: COMT (rs4680), ZDHHC8 (rs175174) and UFD1L (rs5992403). These findings indicate that alterations in the CC volume in children with 22q11DS are associated with cognition and specific genotypes in the 22q11.2 interval.

European Journal of Human Genetics (2012) 20, 1051-1057; doi:10.1038/ejhg.2012.138; published online 4 July 2012

Keywords: chromosome 22q11.2 deletion syndrome; velocardiofacial syndrome; corpus callosum; neurocognition; schizophrenia

\section{INTRODUCTION}

Chromosome 22q11.2 deletion syndrome (22q11DS), also known as velocardiofacial syndrome or DiGeorge syndrome, is associated with a variety of manifestations. ${ }^{1,2}$ Cognitive deficits are seen in the majority $(80-100 \%)$ of individuals with $22 \mathrm{q} 11 \mathrm{DS}^{3}$ with impairments in sustained attention, executive function, memory, visual-spatial perception, ${ }^{4-6}$ along with anxiety disorders, attention deficit disorder and poor social skills $(40-50 \%) .^{7-9}$ A uniquely elevated risk of schizophrenia spectrum disorders (25\%), bipolar disorder $(\sim 5 \%)$ and major depression $(\sim 10 \%)$ occurs in adolescence and young adulthood. ${ }^{10,11}$

Brain MRI studies have shown volumetric changes in multiple cortical and subcortical regions in individuals with 22q11DS that could be related to both cognition and psychoses. ${ }^{12,13-15}$ However, more studies that delineate the neuroanatomical findings in childhood are needed to tease out findings that are specifically associated with cognition.

The genes within a $1.5-\mathrm{Mb}$ interval in the $22 \mathrm{q} 11.2$ deleted region are thought to be critical for the development of the cognitive/neuropsychiatric phenotype in individuals with 22q11DS. ${ }^{16}$ Although undoubtedly haploinsufficiency contributes to these manifestations, the relationship between the deletion and brain development is unclear.

The human corpus callosum (CC) is the largest white matter pathway between the cerebral hemispheres and has a pivotal role in interhemispheric transfer of cognitive information. ${ }^{17}$ The fiber connections between corresponding hemispheric regions are topographically arranged; thus, structural changes in the CC are reflective of changes in the corresponding cortical areas. Diminished connectivity in the CC may be involved in the pathophysiology of many neurodevelopmental disorders ${ }^{18}$ and schizophrenia. ${ }^{19}$

We previously reported that the CC area was larger in children with 22q11DS, compared with typically developing children, ${ }^{20}$ replicated by others. ${ }^{21-23}$ Relatively little is known about the association between the CC size and the neurocognitive/psychiatric manifestations of 22q11DS, with one report of an association between ADHD and smaller CC area $^{21}$ and another of faster enumeration skills with a larger CC. ${ }^{22}$

Our main objective in this study was to examine volumetric differences in the CC in children with 22q11DS in relationship to neuropsychological manifestations and specific functional singlenucleotide polymorphisms (SNPs) within the COMT (rs4680 or

${ }^{1}$ Division of Medical Genetics, Department of Pediatrics, Duke University Medical Center, Durham, NC, USA; ${ }^{2}$ Department of Pediatrics, Wake Forest University Health Sciences, Winston-Salem, NC, USA; ${ }^{3}$ Department of Psychiatry, Harvard Medical School, Boston, MA, USA; ${ }^{4}$ Department of Psychiatry, University of North Carolina at Chapel Hill, Chapel Hill, NC, USA; ${ }^{5}$ Department of Radiology, Duke University Medical Center, Durham, NC, USA; ${ }^{6}$ Department of Radiology, Wake Forest University Health Sciences, Winston-Salem, NC, USA; ${ }^{7}$ Department of Biostatistics, Wake Forest University Health Sciences, Winston-Salem, NC, USA

*Correspondence: Dr V Shashi, Division of Medical Genetics, Department of Pediatrics, Duke University Medical Center, Box 103857, Durham, NC 27710, USA. Tel: +1 919681 2616; Fax: +1 919668 0414; E-mail: Vandana.shashi@duke.edu

Received 4 December 2011; revised 23 May 2012; accepted 31 May 2012; published online 4 July 2012 
$\left.{ }^{\mathrm{Val}} 158^{\mathrm{Met}}\right), Z D H H C 8$ (rs175174), PRODH (rs450046) and UFD1L (rs5992403) genes, within the corresponding 1.5-Mb interval of the $22 \mathrm{q} 11.2$ region. These SNPs were selected based on literature reports that they could be involved in the cognitive/psychiatric manifestations in 22q11DS. ${ }^{24-27}$ Our specific hypotheses derived from our preliminary data and the literature ${ }^{20,28,29}$ were: (a) 22q11DS subjects would have larger regional and total CC volumes, (b) children with 22q11DS would demonstrate an abnormal trajectory of CC volumes in relationship to age, (c) total CC and regional volumes would correlate with several aspects of cognition in the 22q11DS group; the genu volume correlating with executive function and language and the splenium volume with language and (d) within the 22q11DS subjects, there would be differences in CC volumes on the basis of genetic polymorphisms. Because the Met allele of the COMT gene has been reported to be associated with better cognition in individuals with 22q11DS, ${ }^{24,30}$ we postulated that the Met allele would be associated with higher CC volumes.

\section{METHODS}

\section{Demographics}

Participants included 59 patients with 22q11DS and 53 control subjects. A small subset of the subjects (13 in each group) had had area measurements of the CC previously. ${ }^{20}$ The 22q11DS subjects were enrolled into the study at Wake Forest University (WFU) and Duke University Medical Center (DUMC), with IRB approval. The control subjects were recruited through the public school systems and pediatric practices

The 22q11DS and control groups did not differ on gender (Fisher's exact test, $P=0.213)$ or age $(\mathrm{M}=10.46, \mathrm{SD}=2.35$ and $\mathrm{M}=10.84, \mathrm{SD}=1.88$, respectively, $P=0.355$ ). There were significant differences in ethnic composition between the control and 22q11DS groups $\left(X^{2}=14.17, P<0.05\right)$ because recruitment of control subjects represents the community demographic in the area of DUMC, with 28.3\% African-American subjects in the control group and $3.4 \%$ in the 22q11DS group. All the subjects were nonpsychotic.

\section{MRI acquisition}

Brain MRI scans were performed at the two sites of the study.

Site 1 (WFU). T1 Images were acquired at the WFU General Electric (GE, Fairfield, CT, USA) 1.5-T Signa System running 8.4 M4 software, as previously published. ${ }^{14}$

Site 2 (DUMC). Whole Brain T1 Spoiled GRASS images were acquired with a GE 3T scanner (details in Supplementary Materials).

Morphometry of the CC volume using FreeSurfer. The CC was identified using an automated labeling method and divided into five subregions (Figure 1): the anterior sixth (genu), the anterior half minus the anterior sixth (posterior genu), posterior half minus the posterior third (truncus), posterior third minus the posterior one-fourth (anterior splenium) and the posterior

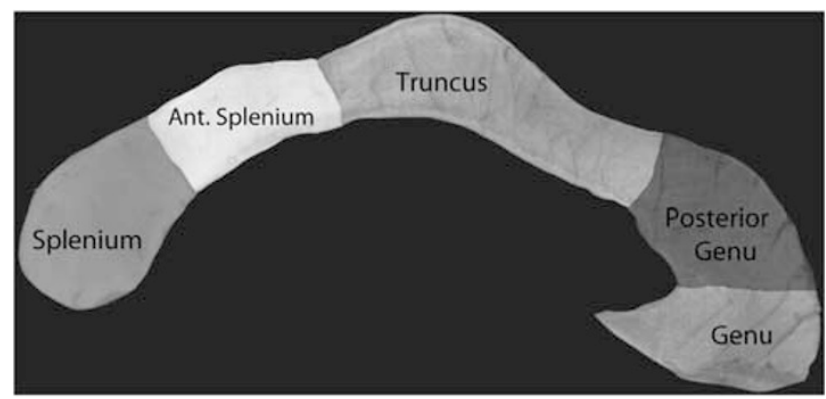

Figure 1 Subregions of the CC that were volumetrically assessed in the 22q11DS and control groups. one-fourth (splenium). The volume of the CC was taken as the sum total of the area of the CC in the mid-sagittal slice and two sagittal slices to the right and left side.

\section{Neuropsychological assessments}

A battery of tests (Table 1) was administered by the same personnel across both medical centers and all testers were blinded to the imaging data. Similarly, the investigators who analyzed the MRI data were blinded to the neuropsychological data or group membership. The Computerized Diagnostic Interview Schedule for Children (C-DISC) ${ }^{31}$ was administered to assess psychiatric diagnoses.

\section{Genotyping studies in the 22q11DS group}

The alleles for functional SNPs in the COMT (rs4680 or Val $158^{\mathrm{Met}}$ ), PRODH (rs450046), UFD1L (rs5992403) and ZDHHC8 (rs175174) were ascertained by SNP mapping, using MassArray and iPLEX assay from Sequenom (San Diego, CA, USA).

\section{Statistical analysis}

We used SPSS (IBM Corporation, Armonk, NY, USA) version 18.0. The data were examined for homogeneity of variance, and parametric tests were performed for variables that satisfied the homogeneity assumption and nonparametric methods for variables that did not. Although MANCOVA algorithms account for multiple comparisons, we performed Bonferroni corrections of the six CC volumes for multiple comparisons. Pearson's correlations were computed to examine the relationship between the six CC volumes and five neurocognitive measures. To assess if the CC volumes were differentially associated with neurocognition in the two groups, a set of linear regression analyses were computed.

\section{RESULTS}

\section{CC volumetric studies}

Site. There were no significant differences in the number of subjects across the two sites (32 subjects with 22q11DS and 32 controls at the WFU site; 27 subjects with 22q11DS and 21 controls at the DUMC site, Fisher's exact test, $P>0.1$ ). There were no significant differences in gender, ethnicity, age or SES at the WFU site. For the Duke site, there were no significant differences in gender, age and SES. Ethnicity was significantly different $\left(\mathrm{X}^{2}=18.3, P<0.01\right)$ due to a larger number of African-Americans in the control group.

Table 1 Neuropsychological tests administered to ascertain specific domains of cognition ${ }^{\mathrm{a}}$

Domains for psychological variables

\begin{tabular}{ll}
\hline Fine Motor & WISC coding \\
Attention & CPT_AX d'prime \\
Language & WISC verbal comprehension \\
Visual & WISC perceptual organization \\
Memory & CVLT list A trials A15
\end{tabular}

Executive function

Working memory WISC working memory

Processing speed WISC processing speed

Set-shifting

Problem solving

WCST perseverative errors/trails B time WCST categories completed

$\begin{array}{ll}\text { Academic } & \\ \text { Reading } & \text { WIAT total reading } \\ \text { Mathematics } & \text { WIAT mathematics }\end{array}$

aAll data are reported as age-based standard scores, except for CPT_AX d'prime, which is reported as a raw score. 
Site 1 (WFU): MANCOVA with age, gender and intracranial volume (ICV) as covariates, group as the independent variable and the total $\mathrm{CC}$ and the five subregional volumes as dependent variables revealed that there were significant differences between the 22q11DS and control groups on the CC volumes: $\mathrm{F}(5,55)=13.95$, Wilks' Lambda $=0.441, P<0.001$. Individual ANCOVAs showed that the Genu $\mathrm{F}(1,59)=32.4, P<0.001, \eta \mathrm{p}^{2}=0.35$, splenium $\mathrm{F}(1,59)=$ 30.63, $P<0.001, \eta \mathrm{p}^{2}=0.34$ and total $\mathrm{CC} \mathrm{F}(1,59)=13.02, P<0.01$, $\eta \mathrm{p}^{2}=0.18$ had significantly larger volumes in the 22q11DS group, relative to the control group with medium to large effect sizes in all instances. There was a trend for the truncus to be smaller in the 22q11DS group $\mathrm{F}(1,59)=3.21, P=0.07, \eta \mathrm{p}^{2}=0.05$ (small effect size). Because Levene's test of homogeneity of variance showed a significant difference between the 22q11DS and control groups for volumes in the splenium and the total CC, we applied the nonparametric Mann-Whitney $U$-test for independent samples to these volumes, with a significant difference between the 22q11DS and control groups for the total CC volume $(P<0.05)$ and the splenium $(P<0.001)$.

Site 2 (Duke): MANCOVA with age, gender and ICV as covariates demonstrated that there were significant differences between the 22q11DS and control groups in the CC volumes: $F(5,48)=5.792$, Wilks' Lambda $=0.587, P=<0.001, \eta \mathrm{p}^{2}=0.42$. Individual ANCOVAs showed that the genu was significantly larger $\mathrm{F}(1,48)=5.52, P<0.05$ with a medium effect size (partial eta-squared $=0.11$ ), whereas the truncus was significantly smaller in the 22q11DS group, relative to controls $\mathrm{F}(1,48)=8.07, P<0.01$ with a medium effect size (partial eta-squared $=0.15$ ). The splenium and total $\mathrm{CC}$ volume were not significantly increased in this cohort, although the direction of results for the splenium (increased volume in children with 22q11DS) was similar to that seen in site 1 .

Pooled data analyses. As the results across both sites were in the same direction, we pooled the CC volumetric data from the sites with Z-transformation, adapting a considered practice to account for the variability in the scanners of different field strength. ${ }^{32,33}$ This increased the number of subjects to 112 . No significant differences in ICV were found between the 22q11DS and control groups. We examined the combined data set for effects of the site of the MRI on the CC volumes and found no site effects or site by group interactions.

\section{Correlations of CC volumes and demographic data}

22q11DS group. On Pearson's correlations, significant correlations were found between ICV and genu, splenium and total CC. There were no differences in CC volumes between males and females. There were no significant correlations between CC volumes and ethnicity or parental SES in the 22q11DS group. When we examined the correlation between age and the CC volumes in the 22q11DS group, there were none, in contrast to the control group (Figure 2).

Control group. ICV was significantly positively correlated with volumes of the all subregions of the CC and the total CC. In contrast to the 22q11DS group, age was significantly positively correlated with the volume of the splenium (Figure 3 ) and anterior splenium. Gender in the control group was not significantly associated with higher volumes in the total CC or the subregions. There were no significant correlations between CC volumes and ethnicity or SES in the control group. Because age, gender and ICV were correlated with CC volumes in one or both groups, we used these as covariates in the MANCOVA

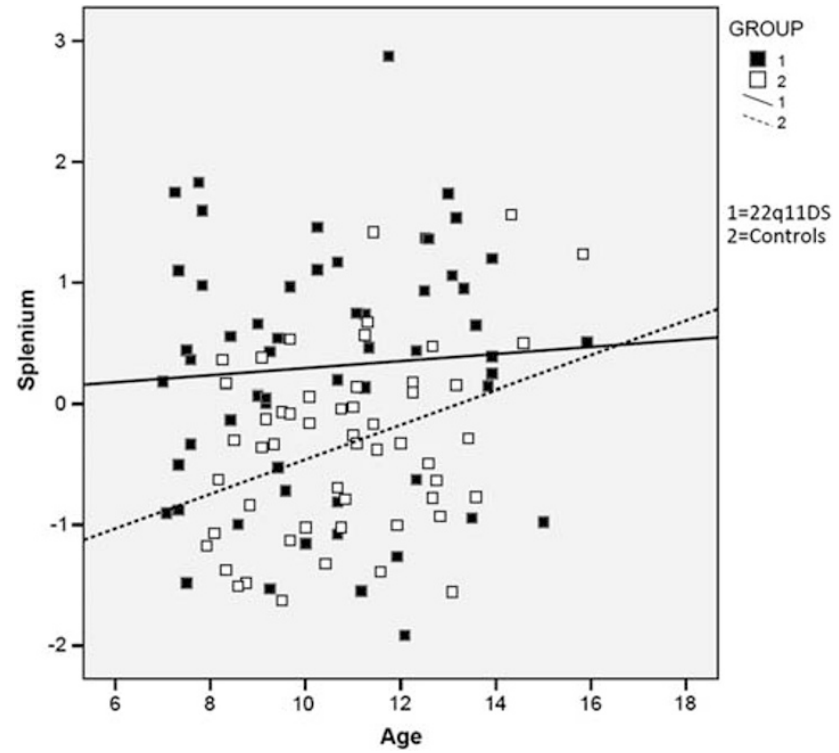

Figure 2 Age and splenium correlations in the 22q11DS and control groups, demonstrating the correlation of age and splenium volume in the control group. The splenium volumes represent standardized Z-transformed scores. Note the lack of an age-related increase in the volume of the splenium in the 22 q11DS group $\left(R^{2}=0.004\right)$ relative to the control group $\left(R^{2}=0.127\right)$.

analyses of CC volumes. Ethnicity and parental SES were not used as covariates, because they did not correlate with CC volumes in either group. Although there was no significant site by group interaction for the CC volumes, we used site of the MRI also as a covariate in the multivariate analyses because we are combining CC volumes across scanners of different field strengths.

\section{CC volumetric differences between 22q11DS and control groups}

On MANCOVA with group as the independent variable and age, gender, ICV and site of MRI as covariates and subregions of the CC and total CC volume as dependent variables, significant differences were evident between the 22q11DS and control groups, $F(5,112)=$ 16.93, Wilks' lambda $=0.546, P<0.001$. Interrogative ANCOVA analyses showed that the genu, splenium and the total CC volumes were larger and the truncus volume was significantly smaller in the 22q11DS group (Table 2). After application of the Bonferroni correction for the six CC volume comparisons, the increase in genu and splenium volumes in the 22q11DS group remained significant; the total CC volume increase and the decrease in the truncus volumes were not significant.

Levene's test of homogeneity with the combined MRI data also demonstrated that there were significant differences $(P<0.05)$ between the 22q11DS and control groups for the splenium and total CC volumes and thus a Mann-Whitney $U$-test was additionally performed; the splenium volume remained significantly different in the 22q11DS group $(P=0.001)$ and the total CC volume narrowly missed significance between the two groups $(P=0.053)$.

Because medication can be associated with volumetric changes, we reran the MANCOVA with any medication for psychiatric diagnoses (ADHD and anxiety/depression) as a covariate and the results were unchanged, with the same significant volumetric differences in the total CC, genu, splenium and truncus between the 22q11DS and control groups. We also examined left and right cerebral 

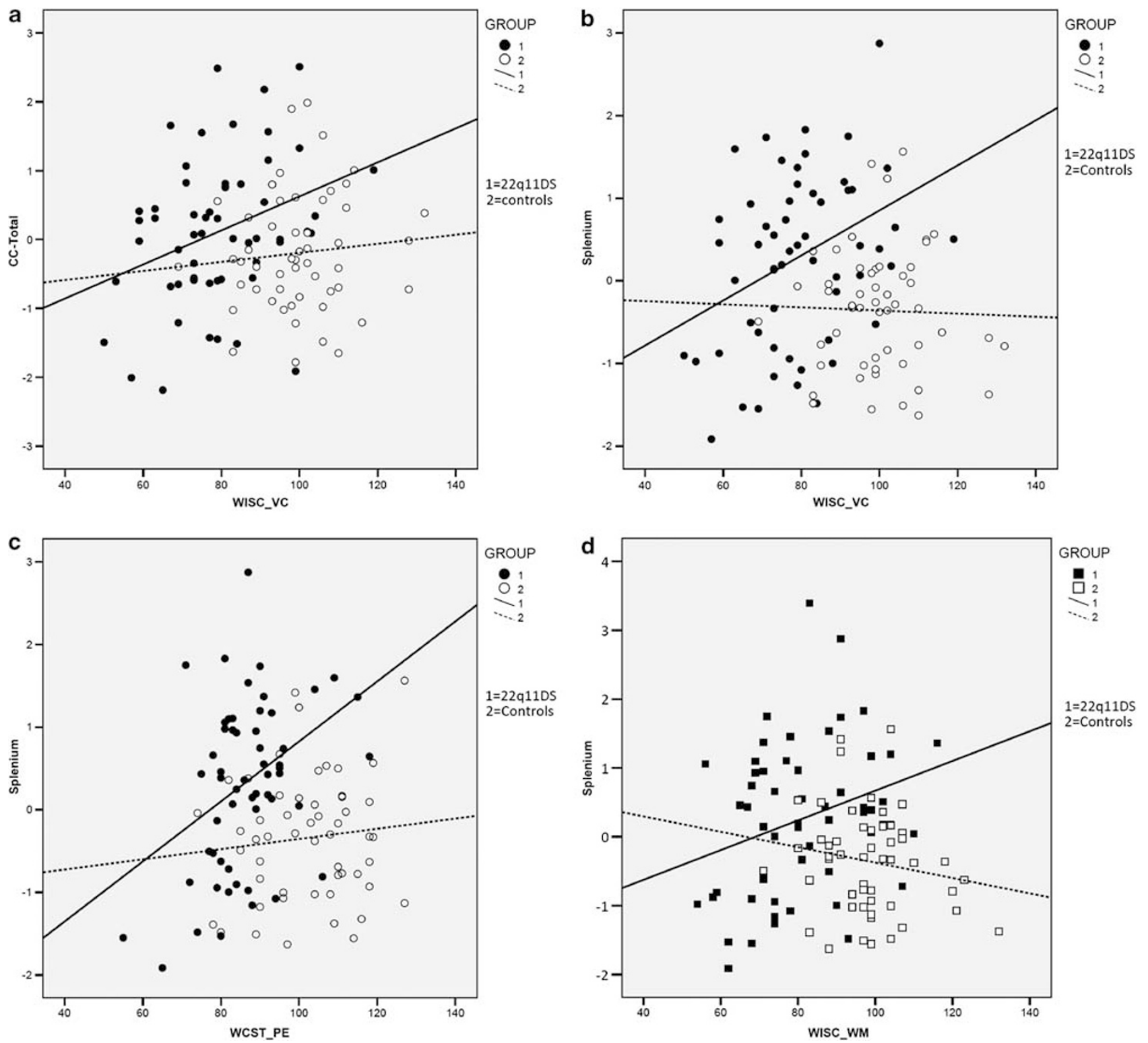

Figure 3 Children with 22q11DS demonstrate that significantly better language abilities are associated with larger total CC and splenium volumes (a, b) and perseverative errors on the WCST with splenium volumes (c) and working memory with larger total CC volumes (d).These significant correlations are absent in the control group.

Table 2 Individual ANCOVAS for the differences in the CC volumes between the 22q11DS and control groups with ICV, site of MRI, age and gender as covariates (22q11DS $=59$ and controls $=53$ )

\begin{tabular}{|c|c|c|c|c|c|c|c|}
\hline CC region & $\begin{array}{c}22 q 11 D S \text { subjects } \\
\text { mean (SD) }\end{array}$ & $\begin{array}{c}\text { Control subjects } \\
\text { mean (SD) }\end{array}$ & $\begin{array}{l}\text { Df and } \\
\text { number }\end{array}$ & $F$ & $\begin{array}{c}\text { Effect size } \\
\text { (partial eta-squared) }\end{array}$ & $\begin{array}{c}\text { Site } \\
\text { effect (F) }\end{array}$ & $\begin{array}{c}\text { Site } \times \text { group } \\
\text { interaction }(F)\end{array}$ \\
\hline Genu & $0.3949(0.9386)$ & $-0.4396(0.8819)$ & 1,112 & $33.03^{* *}$ & 0.23 & 0.34 & 2.71 \\
\hline Truncus & $-0.3187(1.040)$ & $0.3548(0.8265)$ & 1,112 & $10.55^{*}$ & 0.09 & 0.02 & 0.83 \\
\hline Anterior Splenium & $-0.0368(1.088)$ & $0.0410(0.9001)$ & 1,112 & 0.04 & 0.00 & 0.00 & 0.88 \\
\hline Splenium & $0.3043(1.097)$ & $-0.3388(0.7547)$ & 1,112 & $19.21^{* *}$ & 0.15 & 0.00 & 3.38 \\
\hline
\end{tabular}

Degrees of freedom and number of scans are 1 and 112, respectively.

Medium (0.09) and large (0.25) effect sizes are in bold.

${ }^{*} P>0.01$.

$* * P<0.001$. 
hemisphere white-matter volumes in the 22q11DS and control groups with the combined data set and did not see a significant difference.

\section{CC and cognition/psychiatric diagnoses correlations}

Within the 22q11DS group, significant positive correlations were seen between the splenium volume and language, executive function and set-shifting (Table 3 and Figure 3). The genu and total CC volumes were positively associated with set-shifting. No negative correlations between the CC volumes and neurocognitive measures were evident. There were no significant differences in CC volumes in children with 22q11DS who had anxiety disorders and those that did not.

We saw no correlations between language or working memory, anxiety disorders and CC volumes in the control group, but the control group had a correlation between anterior splenium and shorter trails $\mathrm{B}$ time $(r=0.34, P<0.05)$.

Table 3 Pearson's correlations of CC volumes with selected neurocognitive measures in children with 22q11DS $(n=59$, trails B time data available only for 25 subjects)

\begin{tabular}{|c|c|c|c|c|c|}
\hline CC region & $\begin{array}{c}\text { Verbal } \\
\text { comprehension }\end{array}$ & $\begin{array}{l}\text { Working } \\
\text { memory }\end{array}$ & $\begin{array}{l}\text { Trails } \\
\text { B time }\end{array}$ & $\begin{array}{c}\text { WCST perseverative } \\
\text { errors }\end{array}$ & $\begin{array}{c}\text { WIAT math } \\
\text { score }\end{array}$ \\
\hline Genu & 0.20 & 0.17 & $-0.46^{*}$ & 0.11 & 0.09 \\
\hline $\begin{array}{l}\text { Posterior } \\
\text { Genu }\end{array}$ & 0.24 & 0.06 & -0.32 & 0.13 & 0.10 \\
\hline Truncus & 0.21 & 0.01 & -0.34 & 0.10 & 0.07 \\
\hline $\begin{array}{l}\text { Anterior } \\
\text { Splenium }\end{array}$ & 0.21 & 0.10 & -0.27 & 0.24 & 0.03 \\
\hline Splenium & $0.35^{* *}$ & $0.30 *$ & $-0.46^{*}$ & $0.36^{* *}$ & $0.28^{*}$ \\
\hline Total CC & $0.33^{*}$ & $0.28 *$ & $-0.50^{*}$ & $0.26^{*}$ & 0.17 \\
\hline
\end{tabular}

Negative correlation with trails B time indicated that increased CC volumes were associated with a shorter time required to complete the task, so if effect these are positive correlations with set-shifting.

Magnitude of correlation: $0.1=$ small, $0.3=$ medium and $0.5=$ large. Medium and large effect sizes are in bold.

${ }^{*} P<0.05$.

${ }^{* *} P<0.01$.
Linear regression analyses

The 22q11DS group membership and a larger splenium volume accounted for a significant variance in working memory and mathematic performance; 22q11DS group membership and a larger splenium volume accounted for a significant variance in set-shifting (Table 4).

\section{CC volumes and genotype relationships}

For the rs4680 polymorphism, the MANCOVA was significant, $\mathrm{F}(5,48)=2.5$, Wilks' lambda $=0.80, P<0.05$. On the interrogative ANCOVAs, the splenium $(\mathrm{F}(1,58)=6.32, P<0.05))$, genu $(\mathrm{F}(1,58)=$ 4.17, $P<0.05)$, posterior genu $(\mathrm{F}(1,58)=7.3, P<0.01)$ and the total $\mathrm{CC}$ volumes $(F(1,58)=9.43, P<0.01)$ were significantly larger in 22q11DS children who had the Met allele (A). The truncus volume was conversely smaller in those with the Met allele $(\mathrm{F}(1,58)=5.79, P<0.05)$.

The MANCOVA for rs175174 was significant $F(5,49)=2.74$, Wilks' lambda $=0.78, P<0.05$. The volumes of the genu $(\mathrm{F}(1,53)=$ 4.82, $P<0.05)$, posterior genu $(\mathrm{F}(1,59)=7.73, P<0.01)$, splenium $(\mathrm{F}(1,59)=6.6, P<0.05)$ and total $\mathrm{CC}(\mathrm{F}(1,59)=9.99, P<0.01)$ were larger with the A allele of rs 175174 and the truncus volume was smaller with the A allele $(\mathrm{F}(1,59)=6.6, P<0.05)$. For rs5992403, the genu $(\mathrm{F}(1,37)=8.81, P<0.01)$ and the total $\mathrm{CC}(\mathrm{F}(1,37)=4.6, P<0.05)$ were larger for the $\mathrm{A}$ allele and the truncus was smaller in those with the A allele $(\mathrm{F}(1,37)=5.3, P<0.05)$ (note that the rs5992403 genotypes were available for 37 individuals with 22q11DS). The SNP rs450046 was not polymorphic enough to enable further testing.

\section{DISCUSSION}

Our study, the first to volumetrically measure the CC in children with 22q11DS, demonstrated that the total CC, genu and splenium are larger, yet there is a lack of an age-related increase in the CC in these children. Previous studies in children with 22q11DS had examined the area of the CC in the mid-sagittal plane, ${ }^{20-22}$ but differences in fiber density within the CC can result in asymmetries that, in turn, can affect area measurements. ${ }^{34}$ Volumetric measurements are less subject to such variances and would thus be more accurate.

Table 4 Linear regression analyses to differentially predict neurocognition as a function of CC subregional volumes in 22q11DS and control groups

\begin{tabular}{|c|c|c|c|c|c|c|c|c|c|c|c|c|}
\hline \multirow[b]{2}{*}{ Criterion } & \multicolumn{3}{|c|}{ Age } & \multicolumn{3}{|c|}{ Group } & \multicolumn{3}{|c|}{ Splenium volume } & \multicolumn{3}{|c|}{ Group $\times$ splenium } \\
\hline & $\beta$ & $\Delta r^{2}$ & $f^{2}$ & $\beta$ & $\Delta r^{2}$ & $f^{2}$ & $\beta$ & $\Delta r^{2}$ & $f^{2}$ & $\beta$ & $\Delta r^{2}$ & $f^{2}$ \\
\hline Language & 0.11 & 0.01 & 0.01 & 0.60 & $0.37^{* *}$ & 0.59 & 0.17 & $0.03^{*}$ & 0.02 & -0.11 & 0.02 & 0.02 \\
\hline Set-shifting time & -0.37 & $0.14^{* *}$ & 0.16 & -0.49 & $0.25^{* * *}$ & 0.40 & -0.23 & $0.05^{*}$ & 0.08 & 0.56 & 0.03 & 0.06 \\
\hline Mathematics & -0.15 & 0.02 & 0.02 & 0.57 & $0.32^{* * *}$ & 0.50 & 0.61 & 0.02 & 0.03 & -0.54 & 0 * & 0.04 \\
\hline Set-shifting & -0.37 & $0.14^{* *}$ & 0.16 & -0.41 & $0.25^{* * *}$ & 0.40 & -0.13 & 0.01 & 0.02 & 0.78 & $0.05^{*}$ & 0.09 \\
\hline Criterion & \multicolumn{3}{|c|}{ Age } & \multicolumn{3}{|c|}{ Group } & \multicolumn{3}{|c|}{ Total CC vol } & \multicolumn{3}{|c|}{ Group $\times$ total CC } \\
\hline Language & 0.11 & 0.01 & 0.01 & 0.60 & $0.37^{* * *}$ & 0.40 & 0.18 & $0.03^{*}$ & 0.05 & -0.26 & 0.01 & 0.01 \\
\hline
\end{tabular}

Medium (0.15) and large (0.35) effect sizes $\left(f^{2}\right)$ are in bold.

${ }^{*} P<0.05$.

$* * P<0.01$.

$* * * P<0.001$. 
The mechanisms that could underlie the larger CC volumes in the 22q11DS group are not exactly known, but would include a larger number of axons, an expansion of the diameter of the axons and/or increased myelination. Although we believe that there is a delay or abnormality of gray matter synaptic pruning in children with 22q11DS, ${ }^{14}$ and pruning occurs also in the white matter, ${ }^{35}$ the CC volume increases are unlikely to be a consequence of delayed pruning, because such increases would be expected to be negatively correlated with cognition; additionally, the cortical white-matter volumes were not increased in our cohort of children with 22q11DS. The positive correlations between CC volumes and cognitive performance in our study support the notion of a compensatory increase in size of the CC.

The 22q11DS group showed a deceleration of CC volume with increasing age, in contrast to the control group, as we hypothesized. It has been found in typical individuals that the CC continues to grow through childhood and into adulthood, in contrast to the age-related decrease in cortical gray matter. ${ }^{36}$ This continued growth of the CC occurs in conjunction with increases in white matter and is believed to be due to an increase in myelination as well as axon size, ${ }^{37}$ with better interhemispheric connectivity and transfer of information. ${ }^{37}$ The lack of an age-related increase in CC volume in children with 22q11DS suggests that the CC may follow an aberrant neurodevelopmental trajectory with increasing age, despite an initial larger CC volume, such that the growth fails to match what would be expected to occur normally with age. This could be due to a plateau in the compensatory drive or a decrease in myelination/fiber size with age. One longitudinal study reported an increase in cranial white matter in children with 22q11DS over time, ${ }^{15}$ but there were no CC measurements in this study or others, ${ }^{38}$ and thus the trajectory of CC development in 22q11DS remains unknown.

The MRI data in this study were obtained from two scanners of different field strengths and were combined, while being cognizant of the potential hazards of combining data from scanners of different field strengths. ${ }^{39,40}$ A recent study asserted that brain-imaging data obtained from scanners of different field strengths and manufacturers had high spatial reproducibility and consistency. ${ }^{32}$ Additionally, published studies have combined structural brain MRI data from multiple scanners, both of different field strength and platforms. ${ }^{32,33}$ Nonetheless, we used site as a covariate in the analyses and found no significant site effects or site by group interactions in the CC volumes.

Our finding of a correlation between higher language skills, working memory and set-shifting and increased CC volumes is likely reflective of better interhemispheric connectivity, leading to a better neurocognition in children with 22q11DS with larger CC volumes, especially because the fibers in the CC are excitatory. ${ }^{41}$ The neurocognitive and volumetric correlations that we observed in this study are consistent with the distribution of function within the $\mathrm{CC}^{17}$ and our hypotheses. The splenium was significantly correlated with language, executive function and mathematics in the 22q11DS group; it is the subregion that is most correlated with intelligence, processing speed, expressive vocabulary and reading speed. ${ }^{28,29}$ In general, in typical individuals, it is thought that the increase in size of the CC occurs in parallel with its function, ${ }^{42}$ lending credence to our deduction that the increase in volume is beneficial to function in children with 22q11DS. The mechanism and significance of the reduced truncus volume in children with 22q11DS is unclear. We did not specifically examine gross motor tasks, fibers for which traverse the truncus.

In comparing our findings with those in other neurodevelopmental disorders, it is evident that the changes in 22q11DS are unique. In children with neurofibromatosis type 1 , all the subregions of the CC are larger, but the enlargement is negatively correlated with cognition. ${ }^{43}$ In Williams syndrome, the CC area is smaller, ${ }^{44}$ and in fetal alcohol syndrome, reduced callosal volumes occur and are correlated with poor visual-motor integration. ${ }^{45}$ In children with autism, a reduction in volumes of the CC has been reported, with a lack of the age-related positive correlation between age and CC volume. ${ }^{18}$ Thus, our findings of the CC volumes are unlikely to be due to a non-specific finding associated with cognitive impairments.

The psychological/psychiatric burden of 22q11DS is considerable, making it imperative that the neural underpinnings be delineated, to be able to implement effective interventions. ${ }^{46}$ The lack of a correlation between anxiety disorders or ADHD and CC volumes in our 22q11DS cohort is intriguing; in part, this could be because emotional disorders, such as anxiety, are mediated by the amygdala, a subcortical structure that would not be expected to be reflected in the CC measurements.

We also compared the findings in this study with that reported in schizophrenia, wherein the CC is smaller, ${ }^{47}$ probably reflective of the 'disconnectivity' of the hemispheres. ${ }^{48}$ The trajectory of the CC volume in individuals with 22q11DS who go on to develop psychoses is worthy of study, especially because there appears to be a deceleration of CC growth with age.

There have been no studies thus far that have examined the relationship between genotypes in the 22q11DS region and CC volumes in children with 22q11DS. We found that the A allele (Met) of the COMT rs4680 ( $\left.{ }^{\mathrm{Val}} 158^{\mathrm{Met}}\right)$ polymorphism was associated with larger volumes in the total CC, genu (as hypothesized), posterior genu and splenium. Studies of correlations between this SNP and cognition/psychopathology in 22q11DS have reported inconsistent results. ${ }^{24,49}$ We postulate that the rs4680 polymorphism has an indirect effect upon cognition by mediating brain volumes.

We found correlations between CC volumes and the SNP rs175174 of the ZDHHC8 gene. ZDHHC8-knockout mice have decreased dendritic spines and glutaminergic synapses were corrected with the introduction of the ZDHHC8 enzyme. ${ }^{27}$ There is controversy as to whether rs175714 is associated with schizophrenia in the general population. ${ }^{50}$ One study in humans with 22q11DS failed to show an association between rs175174 and schizophrenia. ${ }^{51}$ In our study, we did not find an association between rs175174 and neurocognition, but did find that the A allele was associated with larger subregional volumes of the CC. Thus, the A allele may be influencing the volumes of the CC subregions, which in turn provide a differential compensatory mechanism that facilitates neurocognition.

The UFD1L gene regulates ubiquitin-specific proteases; both overexpression and inhibition of these results in programmed cell death. ${ }^{52}$ A significant increase in the frequency of the A allele of rs5992403 in UFD1L was reported in patients with schizophrenia. ${ }^{26}$ In our study, subjects with 22q11DS with the A allele had a larger genu and total CC volume and a lower truncus volume. Intriguingly, with rs4680, rs175174 and rs5992403, the reduction in the truncus volume was associated with the same allele that was correlated with an increase in volumes of the other subregions of the CC; we thus postulate that these SNPs mediate $\mathrm{CC}$ volumes differentially in children with 22q11DS.

\section{Strengths and limitations}

All of our subjects were children, minimizing the confounds of combining children and adults. All our subjects were nonpsychotic and thus the findings are not confounded by psychosis. The limitation is that the scans were obtained from scanners of different field strengths; however, we employed measures to minimize this. 
In conclusion, we have demonstrated that $\mathrm{CC}$ volumetric changes in children with 22q11DS correlate with neurocognition; polymorphisms in the COMT, ZDHHC8 and UFD1L genes may mediate the CC volumes, as hypothesized. Longitudinal studies are needed to examine the morphometry of the CC.

\section{CONFLICT OF INTEREST}

The authors declare no conflict of interest.

\section{ACKNOWLEDGEMENTS}

This work was fully funded by the R01 grant NIMH-R01MH078015 (to VS).

1 Shprintzen RJ, Goldberg RB, Lewin ML et al: A new syndrome involving cleft palate, cardiac anomalies, typical facies, and learning disabilities: velo-cardio-facial syndrome. Cleft Palate J 1978; 15: 56-62.

2 McDonald-McGinn DM, LaRossa D, Goldmuntz E et al: The 22q11.2 deletion: screening, diagnostic workup, and outcome of results; report on 181 patients. Genet Test 1997; 1: 99-108.

3 De Smedt B, Devriendt K, Fryns JP, Vogels A, Gewillig M, Swillen A: Intellectual abilities in a large sample of children with Velo-Cardio-Facial Syndrome: an update. J Intellect Disabil Res 2007; 51: 666-670.

4 Woodin M, Wang PP, Aleman D, Donald-McGinn D, Zackai E, Moss E: Neuropsychological profile of children and adolescents with the 22q11.2 microdeletion. Genet Med 2001; 3: 34-39.

5 Lewandowski KE, Shashi V, Berry PM, Kwapil TR: Schizophrenic-like neurocognitive deficits in children and adolescents with $22 \mathrm{q} 11$ deletion syndrome. Am J Med Genet $B$ Neuropsychiatr Genet 2007; 144: 27-36.

6 Simon TJ: A new account of the neurocognitive foundations of impairments in space, time and number processing in children with chromosome 22q11.2 deletion syndrome. Dev Disabil Res Rev 2008; 14: 52-58.

7 Swillen A, Vogels A, Devriendt K, Fryns JP: Chromosome 22q11 deletion syndrome: update and review of the clinical features, cognitive-behavioral spectrum, and psychiatric complications. Am J Med Genet 2000; 97: 128-135.

8 Kiley-Brabeck K, Sobin C: Social skills and executive function deficits in children with the 22q11 Deletion Syndrome. Appl Neuropsychol 2006; 13: 258-268.

9 Shashi V, Veerapandiyan A, Schoch K et al: Social skills and associated psychopathology in children with chromosome 22q11.2 deletion syndrome: implications for interventions. J Intellect Disabil Res 2011; e-pub ahead of print 31 August 2011; doi:10.1111/j.1365-2788.2011.01477.x.

10 Murphy KC, Jones LA, Owen MJ: High rates of schizophrenia in adults with velocardio-facial syndrome. Arch Gen Psychiatry 1999; 56: 940-945.

11 Papolos DF, Faedda GL, Veit S et al: Bipolar spectrum disorders in patients diagnosed with velo-cardio-facial syndrome: does a hemizygous deletion of chromosome 22q11 result in bipolar affective disorder? Am J Psychiatry 1996; 153: 1541-1547.

12 Tan GM, Arnone D, McIntosh AM, Ebmeier KP: Meta-analysis of magnetic resonance imaging studies in chromosome 22q11.2 deletion syndrome (velocardiofacial syndrome). Schizophr Res 2009; 115: 173-81.

13 Eliez S, Schmitt JE, White CD, Wellis VG, Reiss AL: A quantitative MRI study of posterior fossa development in velocardiofacial syndrome. Biol Psychiatry 2001; 49 : 540-546.

14 Shashi V, Kwapil TR, Kaczorowski J et al: Evidence of gray matter reduction and dysfunction in chromosome 22q11.2 deletion syndrome. Psychiatry Res 2010; 181: 1-8.

15 Kates WR, Antshel KM, Faraone SV et al: Neuroanatomic predictors to prodromal psychosis in velocardiofacial syndrome (22q11.2 deletion syndrome): a longitudinal study. Biol Psychiatry 2011; 69: 945-952.

16 Karayiorgou M, Gogos JA: The molecular genetics of the 22q11-associated schizophrenia. Brain Res Mol Brain Res 2004; 132: 95-104.

17 de Lacoste MC, Kirkpatrick JB, Ross ED: Topography of the human corpus callosum. J Neuropathol Exp Neurol 1985; 44: 578-591.

18 Paul LK: Developmental malformation of the corpus callosum: a review of typical callosal development and examples of developmental disorders with callosal involvement. J Neurodev Disord 2011; 3: 3-27.

19 Patel S, Mahon K, Wellington R, Zhang J, Chaplin W, Szeszko PR: A meta-analysis of diffusion tensor imaging studies of the corpus callosum in schizophrenia. Schizophr Res 2011; 129: 149-155

20 Shashi V, Muddasani S, Santos CC et al: Abnormalities of the corpus callosum in nonpsychotic children with chromosome 22q11 deletion syndrome. Neuroimage 2004; 21: 1399-1406.

21 Antshel KM, Conchelos J, Lanzetta G, Fremont W, Kates WR: Behavior and corpus callosum morphology relationships in velocardiofacial syndrome (22q11.2 deletion syndrome). Psychiatry Res 2005; 138: 235-245
22 Machado AM, Simon TJ, Nguyen V, McDonald-McGinn DM, Zackai EH, Gee JC: Corpus callosum morphology and ventricular size in chromosome 22q11.2 deletion syndrome. Brain Res 2007; 1131: 197-210.

23 Baker K, Chaddock CA, Baldeweg T, Skuse D: Neuroanatomy in adolescents and young adults with 22q11 deletion syndrome: comparison to an IQ-matched group. Neuroimage 2011; 55: 491-499.

24 Shashi V, Keshavan MS, Howard TD et al: Cognitive correlates of a functional COMT polymorphism in children with 22q11.2 deletion syndrome. Clin Genet 2006; 69: 234-238.

25 Raux G, Bumsel E, Hecketsweiler B et al: Involvement of hyperprolinemia in cognitive and psychiatric features of the 22q11 deletion syndrome. Hum Mol Genet 2007; 16: 83-91.

26 De LA, Pasini A, Amati $F$ et al: Association study of a promoter polymorphism of UFD1L gene with schizophrenia. Am J Med Genet 2001; 105: 529-533.

27 Mukai J, Dhilla A, Drew LJ et al: Palmitoylation-dependent neurodevelopmental deficits in a mouse model of 22q11 microdeletion. Nat Neurosci 2008; 11: 1302-10.

28 Luders E, Narr KL, Bilder RM et al: Positive correlations between corpus callosum thickness and intelligence. Neuroimage 2007; 37: 1457-1464.

29 Fryer SL, Frank LR, Spadoni AD et al: Microstructural integrity of the corpus callosum linked with neuropsychological performance in adolescents. Brain Cogn 2008; 67: 225-233.

30 Shashi V, Howard TD, Keshavan MS et al: COMT and anxiety and cognition in children with chromosome 22q11.2 deletion syndrome. Psychiatry Res 2010; 178: 433-436.

31 NIMH-CDISC: Computerized Diagnostic Interview Schedule for Children, 2004.

32 Jovicich J, Czanner S, Han X et al: MRI-derived measurements of human subcortical, ventricular and intracranial brain volumes: reliability effects of scan sessions, acquisition sequences, data analyses, scanner upgrade, scanner vendors and field strengths. Neuroimage 2009; 46: 177-192.

33 Stonnington CM, Tan G, Kloppel S et al: Interpreting scan data acquired from multiple scanners: a study with Alzheimer's disease. Neuroimage 2008; 39: 1180-1185.

34 Aboitiz F: Brain connections: interhemispheric fiber systems and anatomical brain asymmetries in humans. Biol Res 1992; 25: 51-61.

35 Luders E, Thompson PM, Toga AW: The development of the corpus callosum in the healthy human brain. J Neurosci 2010; 30: 10985-10990.

36 Giedd JN, Blumenthal J, Jeffries NO et al: Brain development during childhood and adolescence: a longitudinal MRI study. Nat Neurosci 1999; 2: 861-863.

37 Keshavan MS, Diwadkar VA, DeBellis M et al: Development of the corpus callosum in childhood, adolescence and early adulthood. Life Sci 2002; 70: 1909-1922.

38 Gothelf D, Penniman L, Gu E, Eliez S, Reiss AL: Developmental trajectories of brain structure in adolescents with 22q11.2 deletion syndrome: a longitudinal study. Schizophr Res 2007; 96: 72-81.

39 Jovicich J, Czanner S, Greve D et al: Reliability in multi-site structural MRI studies: effects of gradient non-linearity correction on phantom and human data. Neuroimage 2006; 30: 436-443

40 Han X, Jovicich J, Salat D et al: Reliability of MRI-derived measurements of human cerebral cortical thickness: the effects of field strength, scanner upgrade and manufacturer. Neuroimage 2006; 32: 180-194.

41 Bloom JS, Hynd GW: The role of the corpus callosum in interhemispheric transfer of information: excitation or inhibition? Neuropsychol Rev 2005; 15: 59-71.

42 Lebel C, Walker L, Leemans A, Phillips L, Beaulieu C: Microstructural maturation of the human brain from childhood to adulthood. Neuroimage 2008; 40 : 1044-1055.

43 Moore 3rd BD, Slopis JM, Jackson EF, De Winter AE, Leeds NE: Brain volume in children with neurofibromatosis type 1: relation to neuropsychological status. Neurology 2000; 54: 914-920

44 Luders E, Di Paola M, Tomaiuolo F et al: Callosal morphology in Williams syndrome: a new evaluation of shape and thickness. Neuroreport 2007; 18: 203-207.

45 Sowell ER, Mattson SN, Thompson PM, Jernigan TL, Riley EP, Toga AW: Mapping callosal morphology and cognitive correlates: effects of heavy prenatal alcohol exposure. Neurology 2001; 57: 235-244.

46 Young AS, Shashi V, Schoch K, Kwapil T, Hooper SR: Discordance in diagnoses and treatment of psychiatric disorders in children and adolescents with 22q11.2 deletion syndrome. Asian J Psychiatr 2011; 4: 119-124.

47 Arnone D, McIntosh AM, Tan GM, Ebmeier KP: Meta-analysis of magnetic resonance imaging studies of the corpus callosum in schizophrenia. Schizophr Res 2008; 101: 124-132.

48 Ellison-Wright I, Bullmore E. Meta-analysis of diffusion tensor imaging studies in schizophrenia. Schizophr Res 2009; 108: 3-10.

49 Glaser B, Debbane M, Hinard C et al: No evidence for an effect of COMT Val158Met genotype on executive function in patients with 22q11 deletion syndrome. Am J Psychiatry 2006; 163: 537-539.

50 Mukai J, Liu H, Burt RA et al: Evidence that the gene encoding ZDHHC8 contributes to the risk of schizophrenia. Nat Genet 2004; 36: 725-731.

51 Demily C, Legallic S, Bou J et al: ZDHHC8 single nucleotide polymorphism rs175174 is not associated with psychiatric features of the $22 \mathrm{q} 11$ deletion syndrome or schizophrenia. Psychiatr Genet 2007; 17: 311-312.

52 Yamagishi H, Garg V, Matsuoka R, Thomas T, Srivastava D: A molecular pathway revealing a genetic basis for human cardiac and craniofacial defects. Science 1999; 283: 1158-1161.

Supplementary Information accompanies the paper on European Journal of Human Genetics website (http://www.nature.com/ejhg) 